\title{
Multiple Transitions of the Spin Configuration in Quantum Dots
}

\author{
M. C. Rogge, ${ }^{*}$ C. Fühner, and R. J. Haug \\ Institut für Festkörperphysik, Universität Hannover, Appelstraße 2, D-30167 Hannover, Germany
}

(Received 15 June 2006; published 24 October 2006)

\begin{abstract}
Single electron tunneling is studied in a many electron quantum dot in high magnetic fields. For such a system multiple transitions of the spin configuration are theoretically predicted. With a combination of spin blockade and Kondo effect we are able to detect five regions with different spin configurations. Transitions are induced with changing electron numbers.
\end{abstract}

DOI: 10.1103/PhysRevLett.97.176801

Spin physics in semiconductor quantum dots [1] has pushed the research on nanostructures since quantum dots were suggested as essential elements in quantum computing devices [2]. The spin of a single electron on a quantum dot can act as a qubit and thus great efforts were made to prepare, manipulate, and read the spin on few electron quantum dots. But, in addition, the research on many electron dots has come up with exciting results and new effects dealing with spin. While it is possible to detect the spin orientation on very few electron dots just by identifying energy levels, conclusions about the spin configuration in many electron dots must be drawn indirectly from additional spin dependent effects. A few years ago it was demonstrated that so called spin blockade can be used to read the spin of the tunneling electron in lateral quantum dots in a perpendicular magnetic field [3]. More information about the spin configuration can be obtained when a Kondo effect [4] is observed depending on the spin configuration in Coulomb blocked regions [5,6].

In a perpendicular magnetic field with two Landau levels in the quantum dot (filling factor $4>\nu_{\text {dot }}>2$ ), a shell structure is assumed for the dot with Landau level 0 in the edge and level 1 in the core (see Fig. 1) [7]. An unpolarized spin configuration is expected at the edge of the dot for low electron numbers in high magnetic fields [8]. That means the total spin at the edge of the dot is 0 whenever the electron number is even, and $\frac{1}{2}$ when the electron number is odd: $\left(S_{\text {even }}, S_{\text {odd }}\right)=\left(0, \frac{1}{2}\right)$ with $S_{\text {even }}$ and $S_{\text {odd }}$ the total spin at the edge of the dot for even and odd electron numbers (see Fig. 1). A few years ago multiple transitions of the spin configuration were theoretically predicted and calculated at the $\nu_{\mathrm{dot}}=2$ boundary [9]. The spin polarization should increase with increasing electron number, $\left(0, \frac{1}{2}\right) \rightarrow\left(1, \frac{1}{2}\right) \rightarrow\left(1, \frac{3}{2}\right) \ldots$ These transitions of the spin configuration are related to spin flips in the $\nu_{\text {dot }}<2$ regime when the spin configuration approaches the maximum density droplet. The first two spin configurations $(0$, $\left.\frac{1}{2}\right)$ and $\left(1, \frac{1}{2}\right)$ can be identified with the combination of spin blockade and Kondo effect [10]. While the first transition between these two configurations was observed in a few electron quantum dot [11], experimental evidence for multiple transitions was never found.
PACS numbers: 73.63.Kv, 72.15.Qm, 73.21.La, 73.23.Hk

We close this gap and present measurements of multiple transitions in the magnetoconductance of a many electron quantum dot. We make use of the combination of both spin blockade and Kondo effect at $4>\nu_{\text {dot }}>2$ to identify five regions with different spin configurations. Transitions between these regions are induced with changing electron number as predicted. Nevertheless some observations imply slight deviations from the theoretical picture. Evidence is found for a change of spin configuration back and forth [e.g., $\left.\left(0, \frac{1}{2}\right) \rightarrow\left(1, \frac{1}{2}\right) \rightarrow\left(0, \frac{1}{2}\right)\right]$ instead of a continuously increasing spin polarization. These results, as well as results about the position of the transitions as a function of magnetic field, should affect eventually forthcoming calculations for $\nu_{\mathrm{dot}}>2$.

Our sample [12] is fabricated in split-gate technology on a GaAs/AlGaAs heterostructure with a two-dimensional electron system (2DES) $57 \mathrm{~nm}$ below the surface (see inset of Fig. 2). The electron density is $n=3.7 \times 10^{15} \mathrm{~m}^{-2}$, the mobility is $\mu=130 \mathrm{~m}^{2} / \mathrm{V} \mathrm{s}$. An electronic dot diameter of $\sim 250 \mathrm{~nm}$ yields an electron number of $\sim 180$. Differential conductance measurements were carried out

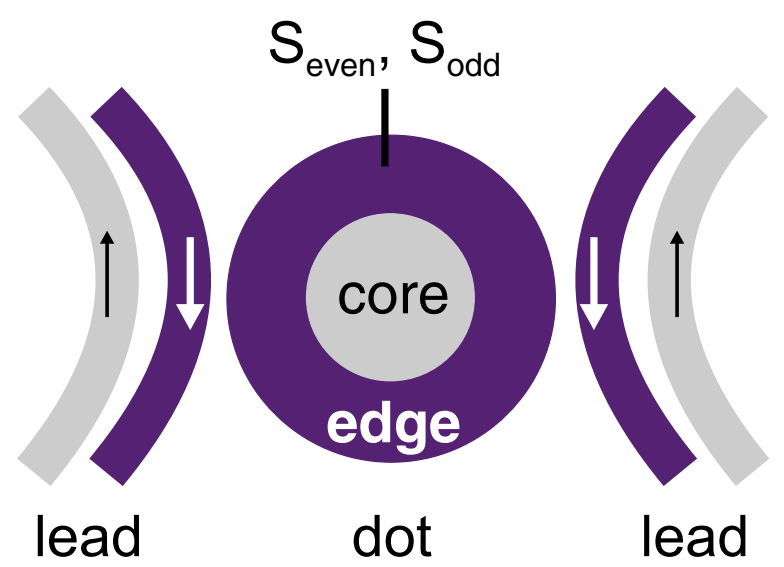

FIG. 1 (color online). Schematic diagram for a lateral quantum dot in a high perpendicular magnetic field. A shell structure with two Landau levels is established in the dot; level 0 at the edge (with total spin $S_{\text {even }}, S_{\text {odd }}$ ) and level 1 in the core. The leads shown are spin polarized due to the spatial separation of edge channels. 


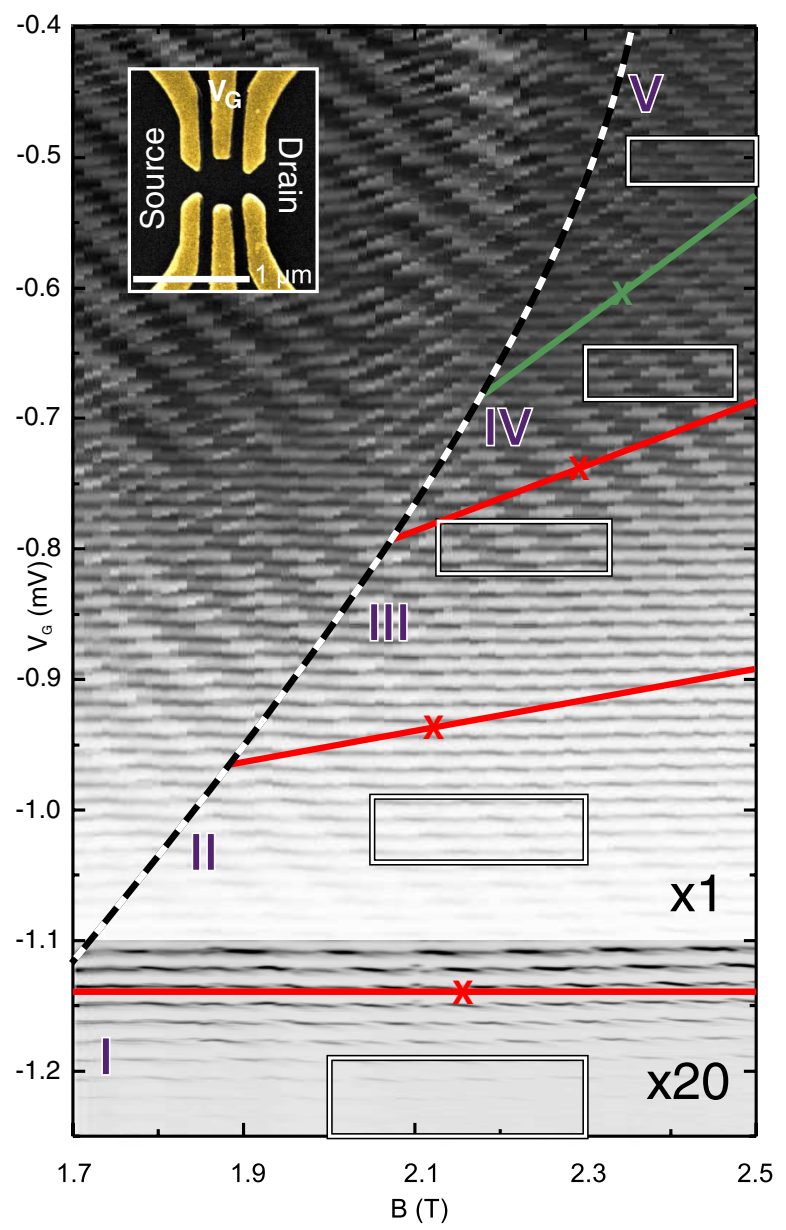

FIG. 2 (color online). Differential conductance $G$ as a function of $B$ and $V_{G}$ (dark for high $G$, white for low $G$ ). On the right of the dashed line a regular chessboard pattern is observed due to Kondo effect and spin blockade. The combination of both effects yields five different regions. The transitions between these regions are marked with solid lines. They are studied in detail later at the positions marked with crosses. Inset: SEM picture of our device.

in a ${ }^{3} \mathrm{He}-{ }^{4} \mathrm{He}$ dilution refrigerator at a base temperature of $20 \mathrm{mK}$ using standard lock-in technique. With the gate voltage $V_{G}$ the dot was tuned in a Kondo regime where signatures of finite Kondo conductance were studied in detail as a function of a magnetic field $B$ applied perpendicular to the sample surface.

Such a measurement is shown in Fig. 2. The differential conductance $G$ is measured as a function of $B$ and $V_{G}$. Many Coulomb peaks are visible, reflecting changes of the electron number by one for each peak (nearly horizontal lines). The depicted range of the gate voltage shows 75 peaks corresponding to a change of the electron number by 75 electrons. In the Coulomb blockade regions between the peaks we find a finite conductance due to the Kondo effect which appears because of second order cotunneling processes. This leads to a chessboard like pattern $[8,13]$ of high and low differential conductance. We concentrate our analysis on the right side of the dashed line were the pattern is most regular. Here in the $4>\nu_{\mathrm{dot}}>2$ regime (the filling factor of the 2DES $\nu_{2 \mathrm{DES}}$ is well above 6 in the magnetic field studied here) only the first Landau level situated at the edge of the dot is involved in the Kondo conductance. Thus the Kondo effect depends on the spin configuration at the edge, which is different for even and for odd electron numbers $\left(S_{\text {even }} \neq S_{\text {odd }}\right)$. Therefore the Kondo effect depends on the parity of the electron number at the edge of the dot (just parity in the following), which is changed either by electron exchange with the leads induced by gate voltage or by electron exchange with the center of the dot induced by the magnetic field.

Apart from the alternating Kondo conductance there is also an alternating peak amplitude of the Coulomb peaks with the same periodicity as the Kondo pattern. This is due to spin blockade [3]. The magnetic field separates the spin polarized edge channels in the leads (see Fig. 1) and thus the tunneling probability and as a consequence the differential conductance becomes spin dependent. Spin-up transport is suppressed compared to spin-down transport. Spin blockade and the Kondo effect can be observed simultaneously in an intermediate field regime [10]. Since the periodicity of both effects is identical, the combination establishes a stable pattern which should not change if the spin configurations for even and odd electron numbers at the edge of the dot do not change. As a consequence, changes of this pattern should reflect changes in the spin configuration at the edge of the dot. If the spin configuration has changed for one parity [e.g. $\left.\left(0, \frac{1}{2}\right) \rightarrow\left(1, \frac{1}{2}\right)\right]$ the pattern is swapped [10].

In our sample we find not less than 5 regions with different combinations of Kondo effect and spin blockade. These are marked with roman numbers I to V in Fig. 2. Therefore we have 4 transitions in between marked with solid lines. Insets in these regions marked with white boxes in Fig. 2 are shown in detail in Fig. 3.

Figure 3(a) shows the differential conductance again as a function of $V_{G}$ and $B$ for all 5 regions. In Fig. 3(b) traces are shown reflecting the amplitude along a Coulomb peak influenced by spin blockade and along the Coulomb valley above showing Kondo conductance.

In region I no Kondo conductance and thus no chessboard pattern is observed since the barriers connecting the dot to the leads are too opaque to allow for second order cotunneling. Nevertheless a clear bimodal pattern of spin blockade is visible.

In region II Kondo conductance sets in (probably not spin- $\frac{1}{2}$ Kondo [12]) and we can observe a clear chessboard pattern. We find that finite Kondo conductance appears above an unsuppressed peak amplitude. Therefore the Kondo effect is connected to a spin down electron added to the edge of the dot.

This pattern is swapped in region III. Here we find the Kondo effect above the suppressed peak amplitude. Thus the Kondo effect is induced by a spin-up electron. 


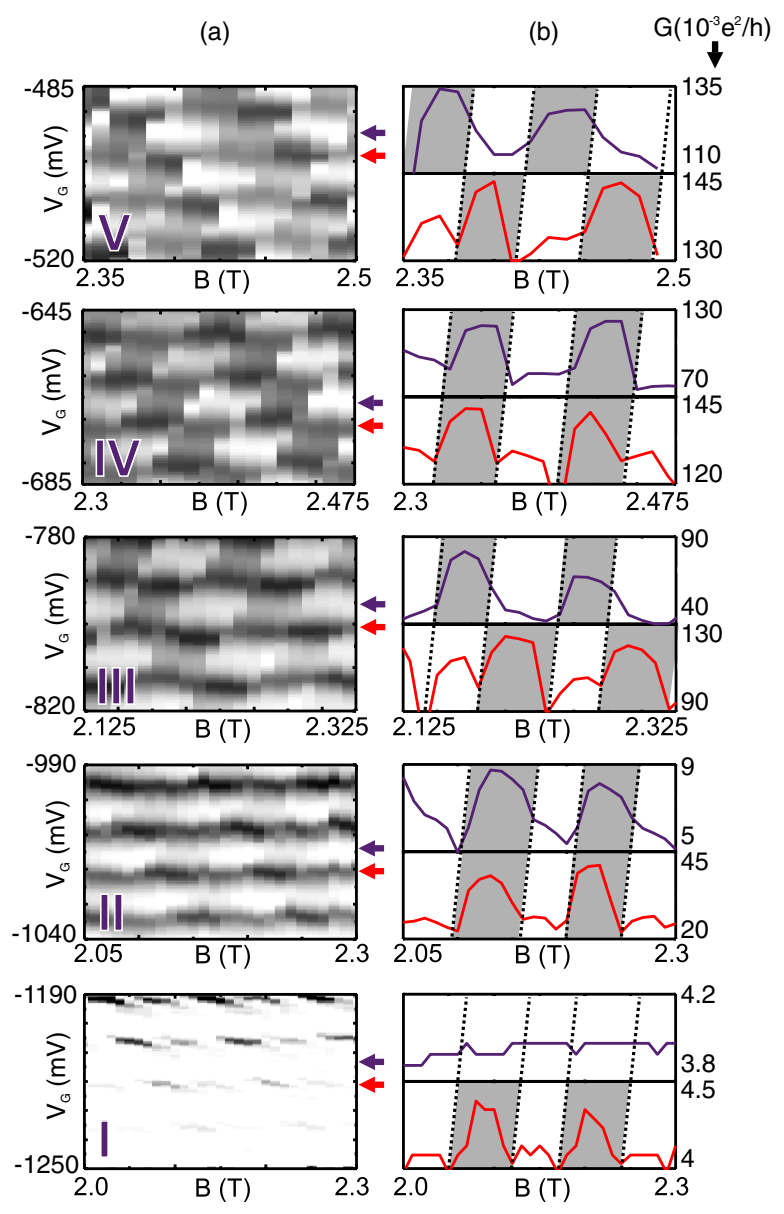

FIG. 3 (color online). (a) Enhanced plots of the insets marked in Fig. 2. In all five regions amplitude modulations of the Coulomb peaks appear due to spin blockade. Apart from region I a clear chessboard pattern is visible due to the Kondo effect as well. The combination of both effects changes from region to region. In II and IV the Kondo effect goes along with a strong amplitude of the Coulomb peak below while the pattern is swapped in III and V. (b) The combination of both effects comes out clearer with the amplitudes of peaks and valleys plotted as a function of $B$. For each region the lower graph shows the spin blockade, the upper graph shows the Kondo effect.

While region IV corresponds to region II, region $\mathrm{V}$ shows again the features of region III.

Now that we have identified these 5 regions we will focus on the details of the transitions between them. This is done exemplarily at the transition from region III to region IV.

In Fig. 4(a) we have plotted the peak amplitude in gray scale as a function of $B$ and the peak number. The resulting plot is comparable to the original measurement without the Coulomb valleys. What is an alternating behavior of peak amplitudes now becomes a pattern of lines with negative slope. A change of this pattern should directly reflect a change in the way electronic spins enter the dot. This in turn corresponds to a change of the spin configuration in (a)

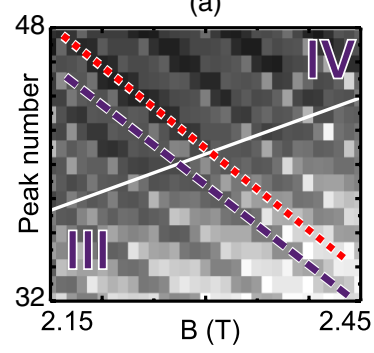

(b)

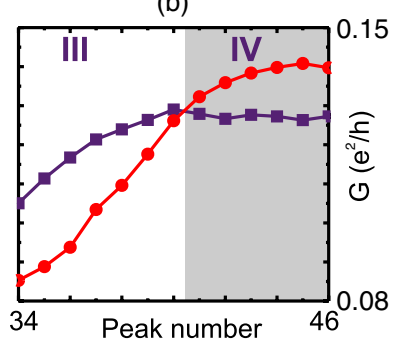

FIG. 4 (color online). (a) Peak amplitude as a function of $B$ and peak number at the transition from III to IV showing only features of spin blockade. What is a bimodal pattern in Fig. 2 now comes out as a pattern of lines with negative slope. At the transition the amplitude of these lines swaps, reflecting a change of the spin configuration. Strong lines become weak and vice versa. (b) Line amplitude along the lines marked dashed and dotted in (a). A crossing appears between peaks 40 and 41 .

the edge of the dot which is exactly what is observed in Fig. 4(a). Lines (dashed) with a strong amplitude below the transition from III to IV (which is marked with a solid line) corresponding to spin-down transport become weak above the transition now showing spin-up transport. In analogy those lines (dotted) with a weak amplitude below the transition become strong above the transition. This is shown in more detail in Fig. 4(b). Traces along lines marked dashed and dotted in (a) are plotted as a function of the peak number. A clear crossing of the peak amplitudes is visible between peak 40 and 41 . Thus the origin for the swap of the total pattern at the transition from region III to IV is identified with the spin blockade signal. The spin filling mechanism has swapped and the spin configuration has changed for one parity. The spin configuration underlying the Kondo effect is not changed as the Kondo effect is not influenced by this transition. Thus the change in spin polarization must have happened for the parity, where no Kondo effect appeared.

Similar analyses were made at all other transitions. The results are shown in Fig. 5. Again two neighboring lines were investigated for each transition as shown before in Fig. 4. But this time for each transition the ratio of the amplitude of these lines is plotted as a function of the peak number. If there was no spin blockade, one would expect both peaks to be equal and thus the ratio would be 1 . We find a swap in the peak ratio at the transitions I to II, II to III, and III to IV which indicates a change in the spin configuration at these transitions. At the first transition no Kondo effect is visible. At the other two transitions no significant influence is found on the Kondo chessboard pattern. As discussed before, this implies that the spin configuration for the parity underlying the Kondo effect is not changed although two swaps of the spin blockade and thus two changes of the spin polarization have occurred (one at II to III, another at III to IV). In our opinion 


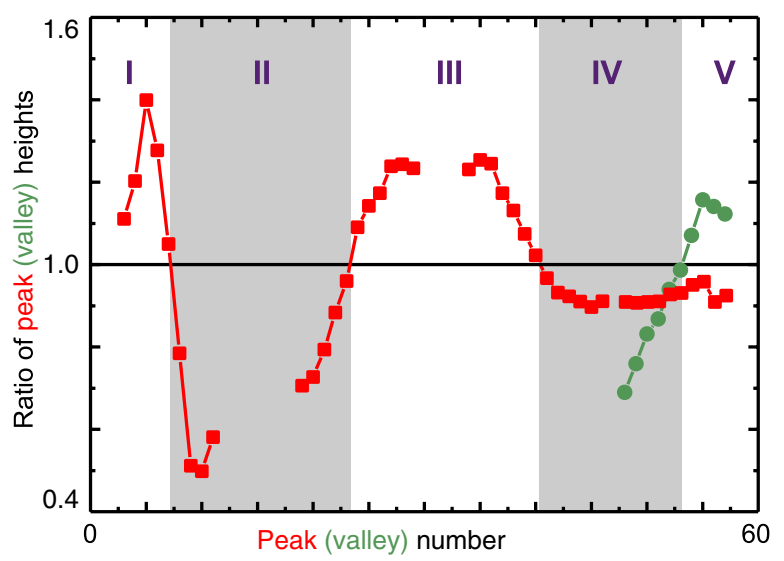

FIG. 5 (color online). For transitions I to IV the ratio of the amplitude of lines similar to those in Fig. 4(b) is plotted as a function of the peak number (squares). Swaps corresponding to changes of the spin configuration appear at the transitions I to II, II to III, and III to IV. No swap was observed at the last transition. Here the Kondo pattern swaps (circles). Please note that the data were not taken at the same magnetic field but at the points marked with crosses in Fig. 2.

this is not possible if the spin polarization would have changed twice in the same direction [e.g., $\left(0, \frac{1}{2}\right) \rightarrow\left(1, \frac{1}{2}\right) \rightarrow$ $\left.\left(1, \frac{3}{2}\right)\right]$. If so, the spin configuration would have changed for both parities which should have affected the Kondo effect. Instead, we believe to have observed a switching of the polarization back and forth [e.g., $\left.\left(0, \frac{1}{2}\right) \rightarrow\left(1, \frac{1}{2}\right) \rightarrow\left(0, \frac{1}{2}\right)\right]$. Thus, only for one parity (the one without the Kondo effect) the configuration is changed.

The last transition from region IV to region $\mathrm{V}$ is different. Here the spin blockade stays unchanged, instead we find a swap of the Kondo pattern marked with circles in Fig. 5. We believe that this is due to a sudden change of the nature of the Kondo effect. This might have happened without changing the spin configurations, as no swap in the spin blockade is observed. However, the slope of the transition line from region IV to region $\mathrm{V}$ marked with a solid line in Fig. 2 implies a different explanation. The slope seems to fit very nicely the slopes of all other transitions. Thus one would expect the same physical origin to be responsible. This could be possible if two swaps at once are assumed changing the spin configuration for both parities [e.g., $\left.\left(0, \frac{1}{2}\right) \rightarrow\left(1, \frac{3}{2}\right)\right]$. In this case the spin blockade is not affected and the change in the nature of the Kondo effect is just due to a new spin configuration.
In conclusion, we have found multiple transitions of the spin configuration in a lateral quantum dot in high magnetic fields as predicted theoretically. These transitions are detected as changes of a combined spin blockade or Kondo effect chessboard pattern. They appear either as a swap of the spin blockade pattern or as a swap in the Kondo pattern. Regardless of the different form of appearance, the slopes of all transitions yield a common origin. They reflect a change of the spin configuration at the edge of the twoLandau-level quantum dot with increasing electron number. In addition, the combination of spin blockade and Kondo effect indicates that a change of the spin polarization back and forth is observed rather than a continuously increasing spin polarization.

This work has been supported by BMBF.

*Electronic address: rogge@nano.uni-hannover.de

[1] L.P. Kouwenhoven, C. M. Marcus, P. L. McEuen, S. Tarucha, R. M. Westerveld, and N.S. Wingreen, in Mesoscopic Electron Transport, edited by L.L. Sohn, L. P. Kouwenhoven, and G. Schön, Series E Vol. 345 (Kluwer, Dordrecht, 1997), p. 105.

[2] D. Loss and D. P. DiVincenzo, Phys. Rev. A 57, 120 (1998).

[3] M. Ciorga, A. S. Sachrajda, P. Hawrylak, C. Gould, P. Zawadzki, S. Jullian, Y. Feng, and Z. Wasilewski, Phys. Rev. B 61, R16315 (2000).

[4] J. Kondo, Prog. Theor. Phys. 32, 37 (1964).

[5] L. I. Glazman and M. E. Raikh, JETP Lett. 47, 452 (1988).

[6] T. K. Ng and P. A. Lee, Phys. Rev. Lett. 61, 1768 (1988).

[7] P. L. McEuen, E. B. Foxman, J. Kinaret, U. Meirav, M. A. Kastner, N. S. Wingreen, and S. J. Wind, Phys. Rev. B 45, 11419 (1992).

[8] M. Stopa, W. G. van der Wiel, S. De Franceschi, S. Tarucha, and L. P. Kouwenhoven, Phys. Rev. Lett. 91, 046601 (2003).

[9] A. Wensauer, M. Korkusinski, and P. Hawrylak, Phys. Rev. B 67, 035325 (2003).

[10] D. Kupidura, M. C. Rogge, M. Reinwald, W. Wegscheider, and R. J. Haug, Phys. Rev. Lett. 96, 046802 (2006).

[11] M. Ciorga, A. Wensauer, M. Pioro-Ladriere, M. Korkusinski, J. Kyriakidis, A.S. Sachrajda, and P. Hawrylak, Phys. Rev. Lett. 88, 256804 (2002).

[12] C. Fühner, U. F. Keyser, R. J. Haug, D. Reuter, and A. D. Wieck, Phys. Rev. B 66, 161305(R) (2002).

[13] M. Keller, U. Wilhelm, J. Schmid, J. Weis, K. von Klitzing, and K. Eberl, Phys. Rev. B 64, 033302 (2001). 